\title{
Performed Protocol Deviation Date Range
}

National Cancer Institute

\section{Source}

National Cancer Institute. Performed Protocol Deviation Date Range. NCI Thesaurus.

Code C93960.

The date and time span specifying when the protocol deviation began and ended. 